\title{
A fatty acid synthase gene (FASN3) from the integument tissue of Rhodnius prolixus contributes to cuticle water loss regulation.
}

\section{E. Moriconi, A. B. Dulbecco, M. P. Juárez, G. M. Calderón-Fernández}

Instituto de Investigaciones Bioquímicas de La Plata (CONICET-UNLP), Facultad de Ciencias Médicas, Universidad Nacional de La Plata, La Plata, Argentina.

Correspondence: Gustavo M. Calderón-Fernández, Instituto de Investigaciones Bioquímicas de La Plata, Facultad de Ciencias Médicas $1^{\circ}$ piso, calles 60 y 120, La Plata, C.P. 1900, Argentina, Tel./fax: + 542214824894 / +54 221 4258988; email: gcalderonf@med.unlp.edu.ar

Running title: Characterisation of $R$. prolixus FASN3 gene

This article has been accepted for publication and undergone full peer review but has not been through the copyediting, typesetting, pagination and proofreading process which may lead to differences between this version and the Version of Record. Please cite this article as doi: $10.1111 /$ imb.12600 


\section{Abstract}

Fatty acid synthase is a multifunctional enzyme involved in the formation of fatty acids. Despite the role of fatty acids in cell signalling and energy metabolism, and as precursors to pheromones and hydrocarbons that waterproof the cuticle, the insect fatty acid synthases have been scarcely studied. Here we perform the molecular characterisation of three fatty acid synthase genes (RPRC000123, RPRC000269 and RPRC002909) in the Chagas disease vector, Rhodnius prolixus. Gene expression screening by RT-qPCR showed that RPRC000123 and RPRC002909 are expressed almost exclusively in the integument tissue whilst $R P R C 000269$ is mostly expressed in the fat body and also in several body organs. Phylogenetic analysis, together with gene expression results, showed that RPRC000269, RPRC002909 and RPRC000123 are orthologues of Drosophila melanogaster FASN1, FASN2 and FASN3 genes, respectively. After RNAi-mediated knockdown of $R P R C 000123$, insects died immediately after moulting to the next developmental stage. However, mortality was prevented by placing the insects under saturated humidity conditions, suggesting that dehydration might play a role in insects' death. Lipid analyses in RPRC000123silenced insects showed reduced amounts of integument fatty acids and methylbranched hydrocarbons, compared to controls.

These data support an important role for FASN3 in the biosynthesis of the precursors to hydrocarbons that waterproof the insect cuticle.

Key words: fatty acid synthase, Chagas disease vector, FASN3 gene, insect integument tissue, cuticle, water loss regulation 


\section{Introduction}

Animal fatty acid synthase (FASN) is a lipogenic megasynthase that catalyses all steps of fatty acid synthesis. It contains seven domains assembled into homodimers, with high conformational flexibility. Regardless of the natural variation in its structural organisation, all organisms use a conserved set of chemical reactions for fatty acid biosynthesis. This process takes place through an iterative series of enzyme reactions in which an acyl chain is extended by two carbon units with each cycle. Initiation occurs after acyl carrier protein (ACP) uploads the acyl groups from acetyl-CoA and malonylCoA units, a reaction catalysed by the malonylacetyl transferase (MAT). A ketoacylsynthase (KS) catalyses a decarboxylative condensation of both acyl-CoA's to an ACP-bound $\beta$-ketoacyl intermediate. The resulting ketone is then modified by sequential action of a ketoreductase $(\mathrm{KR})$, a dehydratase $(\mathrm{DH})$ and an enoyl reductase (ER) yielding an elongated acyl chain which can then be subjected to the same cycle again until the final chain length, usually a 16-carbon fatty acid, is reached. The acyl chain is then released by a thioesterase (TE) (Maier et al., 2008).

Long chain fatty acids (LCFA) participate in a large number of biological processes, as fuel sources, in signal-transduction pathways, cell membrane formation and energy storage, as well as precursors of a variety of other lipid components with numerous functions. The interest on animal and microbial FASN has been renewed, mostly related to cancer research (De Schrijver et al., 2003) and microbial fuel production (Zhou et al., 2016).

Insect FASNs have been less studied; the first insect FASN purification reported was from Drosophila melanogaster fat body, showing the highest specific activity compared to other animal FASNs (De Renobales et al., 1986). This FASN showed an absolute requirement for malonyl-CoA and no activity was observed when methylmalonyl-CoA replaced malonyl-CoA. Fat body FASN is responsible for the biosynthesis of major body storage lipids, mostly deposited as triacylglycerols and transported to different tissues as diacylglycerols via haemolymph (Arrese et al., 2001). 
Evidence that the insect integument, but not the fat body, is the major site of biosynthesis of cuticle LCFA and hydrocarbons $(\mathrm{HC})$, was shown in Triatoma infestans, a triatomine species of high sanitary relevance in South America (Juárez \& Brenner, 1989). This result suggested the possible existence of an additional FASN providing fatty acyl precursors to cuticle $\mathrm{HC}$ and other cuticle lipids. The existence of two insect FASNs, located in the cytosol and microsomal fractions of the integument, were first reported in the cockroach Blattella germanica (Juárez et al., 1992); a microsomal FASN was also reported in T. infestans (Juárez et al., 1996) and in the housefly Musca domestica (Gu et al., 1997). Integument FASN products are used as precursors to straight and methyl-branched $\mathrm{HC}$, and other high molecular weight cuticular components -i.e., fatty alcohols, wax esters- that are essential in waterproofing properties, and in chemical signalling as contact pheromones, among other functions (Blomquist \& Bagnères, 2010; Gibbs, 1998; Hadley, 1994; Juárez \& CalderónFernández, 2007; Wigglesworth, 1945). The biosynthesis of the cuticle lipids takes place in the oenocytes (Wicker-Thomas et al., 2015); these are large subepidermal cells mostly associated with the integument in Rhodnius prolixus (Wigglesworth, 1933), T. infestans (Juárez \& Brenner, 1985) and D. melanogaster (Billeter et al., 2009) or the fat body (Martins et al., 2011).

Three putative FASN genes: CG3523 (FASN1), CG3524 (FASN2), and CG17374 (FASN3) were first reported in the D. melanogaster genome (Parvy et al., 2012). FASN1 is expressed only in the adult fat body, whilst FASN2 and FASN3 are both expressed in adult oenocytes (Chung et al., 2014). The triatomine $R$. prolixus (Hemiptera: Triatominae) is one of the major Chagas disease vectors. With a life cycle and basic biology highly regulated by a blood meal, it has been extensively used as insect model in founder studies of insect physiology, from the discovery of the moulting hormone, to insect growth and reproduction regulation (Wigglesworth, 1933,1975). Three FASN genes (RPRC000123, RPRC000269 and RPRC002909) were recently reported in the R. prolixus genome (Majerowicz et al., 2017) and additionally, two 
FASN gene fragments are present (RPRC000168 and RPRC000923) with most of the enzyme's known domains missing (https://www.vectorbase.org/organisms/rhodniusprolixus) (Mesquita et al., 2015). In T. infestans, a search on an integument transcriptome showed the presence of two integument FASN genes orthologous to RPRC002909 and RPRC000123, respectively (Calderón-Fernández et al., 2017). Evidence that the corresponding FASN activities were differentially affected by chemical insecticides was previously shown in T. infestans (Juárez, 1995). Given the relevance of cuticle lipids to insect fitness, affecting critical steps of their metabolic pathway is expected to alter its major properties as well. Previous studies have shown that altering $\mathrm{HC}$ biosynthesis affected insect survival and desiccation tolerance, together with an enhanced penetration of chemicals through the cuticle (Juárez, 1994,1996). We hypothesize that integument FASNs have a major regulatory role in $\mathrm{HC}$ production as well, being the main provider of the fatty acyl chains necessary to initiate $\mathrm{HC}$ formation. In order to test this hypothesis, we analysed the expression pattern of $R$. prolixus FASN genes in different tissues and performed their phylogenetic analysis. Both RPRC002909 and RPRC000123 were specific of the integument, whereas RPRC000269 was mostly expressed in the fat body. RNA interference (RNAi) experiments showed that $R P R C 000123$ silencing resulted in an abnormal cuticle upon moulting, with a lethal phenotype in usual rearing conditions; silencing the other two FASNs did not affect moulting nor survival. RPRC000123-silenced insects exhibited deficient water balance ability, which was correlated with a significant decrease in the straight and methyl-branched LCFA and methyl-branched HC content in the integument. An increase in the straight chain $\mathrm{HC}$ could be attributed to additional nonintegumental LCFA incorporation into the HC biosynthetic pathway.

\section{Results}

FASN genes expression in R. prolixus tissues and organs 
Screening of FASN transcript expression in different tissues and organs by reverse transcription quantitative PCR (RT-qPCR) showed that $R P R C 000123$ and $R P R C 002909$ were highly expressed in the integument, with a very low expression in the fat body and brain (Fig. 1A, B). RPRC000269 was expressed in all tissues examined, with the highest level detected in the fat body (Fig. 1C). The low expression level of $R P R C 000123$ and $R P R C 002909$ detected in the fat body might be attributed to contamination with the integument, as both tissues are tightly associated, and complete separation is challenging during dissections.

Sequence analysis and phylogeny of R. prolixus FASN genes

Sequence analysis of gene annotations deposited in VectorBase

(https://www.vectorbase.org/Rhodnius_prolixus/Info/Index) showed that RPRC000123 consists of 37 exons spanning through contigs ACPB03006445.1 and

ACPB03006446.1. The full length RPRC000123 cDNA sequence is $6546 \mathrm{bp}$, encoding a predicted protein of 2182 amino acid residues. The annotated protein lacks the MAT and TE domains (Fig. 2A). RPRC002909 consists of 35 exons spanning through contigs ACPB03012543.1 and ACPB03012544.1. RPRC002909 cDNA sequence has a full length of $6171 \mathrm{bp}$, encoding a predicted protein of 2057 amino acid residues. Alignment of the protein sequence with other insect FASNs showed that it contains 6 FASN domains with the TE domain missing. In addition, the protein has two predicted transmembrane domains between the amino acid residues 1493 and 1595 (Fig. 2B). $R P R C 000269$ has 37 exons and is included in the contig ACPB03012542.1. The full length RPRC000269 cDNA sequence is $6165 \mathrm{bp}$ and encodes a predicted protein of 2055 amino acid residues. The protein sequence lacks the DH, ACP and TE domains (Fig. 2C) however, the latter domain is annotated as a different gene (RPRC000391) placed in the adjacent contig (ACPB03012541.1). A phylogenetic analysis of the $R$. prolixus FASN genes showed that they are segregated into two different clades, here named as Clade I and Clade II. 
$R P R C 000269$ and $R P R C 002909$ were included in a small cluster together with FASN genes of other hematophagous hemipterans ( $T$. infestans and Cimex lectularius). Clade I includes this cluster together with other FASN genes of several insect species including D. melanogaster (FASNs 1 and 2), the honey bee Apis mellifera, the cockroach B. germanica and the hemipteran aphid Acyrthosiphon pisum. RPRC000123 was grouped with the FASN3 genes of $D$. melanogaster and $T$. infestans, and with other insect FASN genes forming the Clade II, distantly related to Clade I. Interestingly, the non-insect sequences used as outgroups (FASN genes of humans, the spider Stegodyphus mimosarum, the nematode Caenorhabditis elegans, the crustacean Penaeus vannamei, and the acari Ixodes scapularis) showed a closer relationship to Clade I than to Clade II (Fig. 3).

\section{Effect of FASN genes silencing on insect moulting and survival} In order to silence the expression of the RPRC000123, RPRC000269 and $R P R C 002909$ genes, fourth-stage nymphs were injected with the corresponding double-stranded RNA (dsRNA) synthetized for each gene (dsRPRC000123, dsRPRC000269 or dsRPRC002909). A control insect group was injected with dsRNA from the control gene provided with the silencing kit (dsControl). Insects were maintained under our standard insectary conditions $\left(27^{\circ} \mathrm{C}, 45 \%\right.$ relative humidity $\left.(\mathrm{RH})\right)$. Gene silencing efficiency was measured by RT-qPCR 8 days post injection, resulting in a significant reduction of FASN gene expression (RPRC000123, 94.4\% $\pm 2.9 \%$; RPRC000269, 95\% $\pm 1.2 \% ; R P R C 002909,87.5 \% \pm 5.9 \%)$. Nymphs treated with dsRPRC000269 or dsRPRC002909 moulted to fifth nymph stage and survived normally, similarly as those injected with dsControl. In contrast, insects treated with dsRPRC000123 died immediately after moulting, without concluding the sclerotization and darkening of their cuticle (Fig. 4). 
In order to address whether the lethality resulting from silencing the $R P R C 000123$ gene is correlated to alterations in the FASN products and major derived end-products, the integument LCFA and HC of dsRPRC000123-injected fourth stage nymphs were analysed by capillary gas chromatography coupled to mass spectrometry (CGC-MS), and compared to those of dsControl-injected nymphs. The total amount of LCFA was significantly lower in the RPRC000123-silenced insects (34.04 $\pm 3.55 \mu \mathrm{g} /$ integument) than in the controls $(53.26 \pm 2.44 \mu \mathrm{g} /$ integument) (Fig. 5A). The analysis of the LCFA fractions showed that the straight chains, accounting for the $99.20 \%$ of the total LCFA content, diminished significantly in the RPRC000123-silenced insects (33.93 \pm 3.54 $\mu \mathrm{g} /$ integument) compared to the controls (52.85 $\pm 2.44 \mu \mathrm{g} /$ integument) (Fig. 5B). The methyl-branched fraction, although representing $0.80 \%$ of the total LCFA components, showed a major reduction in the $R P R C 000123$-silenced insects $(0.11 \pm 0.01$ $\mu \mathrm{g} /$ integument) compared to the controls $(0.41 \pm 0.05 \mu \mathrm{g} /$ integument) (Fig. $5 \mathrm{C})$. The amount of integument total $\mathrm{HC}$ diminished significantly in the RPRC000123silenced nymphs (189.93 $\pm 9.22 \mathrm{ng} /$ integument) compared to the control nymphs (248.02 $\pm 8.30 \mathrm{ng} /$ integument) (Fig. 6A). A significant reduction of the methyl-branched $\mathrm{HC}$ amount in the RPRC000123-silenced nymphs (113.06 $\pm 6.06 \mathrm{ng} /$ integument) compared to the control samples ( $210.87 \pm 4.82 \mathrm{ng} /$ integument) explained the total HC decrease (Fig. 6B). Remarkably, straight chain HC amount was significantly higher in the $R P R C 000123$-silenced nymphs $(114.74 \pm 4.33 \mathrm{ng} /$ integument) than in the controls (50.72 \pm 3.94 ng/integument) (Fig. 6C).

\section{High humidity rescue bioassay}

A bioassay was performed to test whether the post-moulting mortality observed in RPRC000123-silenced nymphs (Fig. 4 and 7A) could be attributed to a defective cuticle water barrier due to reduced amounts of cuticle HC. Both dsRPRC000123- and dsControl-injected fourth-stage nymphs were placed into a humid chamber $(96 \% \mathrm{RH}$, $\left.27^{\circ} \mathrm{C}\right) 3$ days prior to their expected moult to the fifth nymph stage. After insects 
successfully moulted, all RPRC000123-silenced nymphs survived for about 48-72 h under these conditions, similarly to control nymphs (Fig. 7B). After that period, RPRC000123-silenced nymphs died even though maintained in a high humidity environment. Differently, all the control insects survived.

\section{Discussion}

Insect's FASNs are responsible for the biosynthesis of LCFA, which are essential not only for the energy metabolism but also as precursors of the very long chain lipids that waterproof the insect cuticle and participate as pheromones in chemical communication, among other relevant functions (Wicker-Thomas et al., 2015). In this sense, insect FASN genes seem to have undergone a process of specialization through gene expansion and tissue-specific expression. The gene expression results showed that RPRC000123 and RPRC002909 are almost exclusively expressed in the integument whilst RPRC000269 is widely expressed in most tissues, but most predominantly in the fat body (Fig. 1). These results give support to early studies performed in the triatomine $T$. infestans, which proposed the activity of different FASNs based on the existence of two pools of fatty acids derived either from the fat body or the integument (Juárez \& Brenner, 1989).

Rhodnius' FASN genes have distinct domains missing (Fig. 2), a feature previously noticed in several insect FASN genes (Finck et al., 2016). As these are very large genes, usually occupying two contigs with sequencing gaps between them, the lack of some domains in these megasynthases is probably due to failures in the genome assembly and/or a wrong gene annotation. In fact, our study shows a fully functional FASN gene despite the absence of the MAT and TE domains in the gene annotation (Fig. 2). Another genome feature suggesting some failures in the gene annotations is the presence of a TE domain upstream to $R P R C 000269$, which was annotated as a different gene (RPRC000391) (Mesquita et al., 2015). An interesting feature of the gene $R P R C 002909$ is the presence of two transmembrane domains on the second half 
of the protein sequence. These domains together with its integument localization allow suggesting that the FASN encoded by this gene might be the orthologue of the FASNs found in the integument microsomal fraction of B. germanica, M. domestica and $T$. infestans in early biochemical studies (Gu et al., 1997; Juárez et al., 1992, 1996). The FASN encoded by RPRC000123 would be the orthologue of the FASNs that in the same studies showed activity in the integument cytosolic fraction. These biochemical studies also showed that both FASNs have the ability to incorporate methylmalonylCoA but showed different activities and primer preference, also different from the fat body FASN (Juárez et al., 1992). The major difference between both integumental FASNs in B. germanica appeared to be related to the turnover of the enzyme-bound intermediates, rather than to differential binding or affinity for malonyl or methylmalonylCoA as supported by the higher specific activity with methylmalonyl-CoA and the much higher ability to incorporate methylmalonyl-CoA by the microsomal FASN. However the amount of the soluble enzyme was much larger than that of the microsomal enzyme (Gu et al., 1993; Juárez et al., 1992). The phylogenetic analysis (Fig. 3) showed that insect FASN genes form two distinct clades, suggesting gene duplication from an ancestral unique gene during insect evolution. Differently, Finck et al. (2016) proposed the existence of three FASN clusters, based on the domains structure and organisation. RPRC000269 and RPRC002909, the orthologues of the D. melanogaster FASN1 and FASN2, are closely related to FASN1 from human and other animal groups. Differently, RPRC000123 seem to form an insect-specific clade together with D. melanogaster FASN3 and other insect FASNs. RPRC000269 and RPRC002909 show high similarity (Fig. 3) and are present in tandem in the genome, suggesting they are derived from a gene duplication event. Interestingly, FASN genes in tandem can be found in several other insects such as D. melanogaster (CG3523 and CG3524, http://flybase.org/), T. castaneum (TC015337 and TC015339, http://beetlebase.org/) the mosquito A. gambiae (AGAP028049 and AGAP008468, https://www.vectorbase.org/organisms/anopheles-gambiae), among others. In addition 
to the three FASN genes, R. prolixus has two gene fragments (RPRC000168 and $R P R C 000923)$ with moderate similarity (36-39\%) to $R P R C 000269$ and $R P R C 002909$. The existence of FASN copies in tandem and gene fragments, as well as the larger number of FASN genes in insect genomes compared to other animal groups, suggests that gene expansion played a major role in the evolution of these genes in insects (Finck et al., 2016; Majerowicz et al., 2017). This process generated different genes also expressed in different tissues- that might acquire distinct roles in the insect physiology and metabolism.

To date, the function of insect FASN genes was thoroughly studied only in some Drosophila species (Parvy et al., 2012; Chung et al., 2014; Wicker-Thomas et al., 2015) . More recently, Tan et al. (2017) showed that FASN silencing suppress lipid accumulation, affecting stress tolerance and water content in Colaphellus bowringi female beetles undergoing diapause. In Drosophila serrata, RNAi-mediated knockdown of the FASN3 using an oenocyte-specific driver was lethal, whilst knockdown of the FASN2 led to a significant reduction of the cuticle methyl-branched $\mathrm{HC}$, associated to a decrease in the desiccation resistance (Chung et al., 2014). Using the same approach, the knockdown of the FASN3 in D. melanogaster had no effect in the cuticle HC content but led to a moderate decrease in the desiccation resistance whilst knockdown of the FASN2 caused a reduction of the methyl-branched $\mathrm{HC}$ amount but was not related to the desiccation resistance (Wicker-Thomas et al., 2015). Taking together, these results show that the function of the FASN genes can be different even in closely related species.

Our results in $R$. prolixus revealed that $R P R C 000123$, the orthologue of the Drosophila FASN3, is involved in the waterproofing properties of the cuticle. RNAi-mediated knockdown of RPRC000269 and RPRC002909 had no effect on the moulting and survival of the nymphs reared under the standard insectary conditions $\left(27^{\circ} \mathrm{C}, 45 \% \mathrm{RH}\right)$. However, knockdown of RPRC000123 triggered insect's death as soon as they moulted (Fig. 4). When the RPRC000123-silenced nymphs were exposed to saturated 
humidity conditions $(96 \% \mathrm{RH})$, the insects survived for about $48-72$ hs after moult (Fig. 7), suggesting that high humidity conditions prevented lethal desiccation.

These results pointed out that a reduced lipid content in the newly formed cuticle of $R P R C 000123$-silenced insects might enable a rapid water loss through the cuticle as soon as they emerged from the old exuviae, finally leading to their death. The analysis of the FASN products showed that RPRC000123 silencing resulted in a significant reduction of the integument total LCFA (Fig. 5A). Both straight and methyl-branched components were significantly diminished (Fig. 5B, C), indicating that the FASN encoded by this gene is involved in the biosynthesis of both chains by accepting either malonyl-CoA or methylmalonyl-CoA as elongating unit. FASN products are the expected substrate for elongation reactions to provide the precursor to straight and methyl-branched HC. Therefore, RPRC000123 silencing also affected the amount of the integument $\mathrm{HC}$ (Fig. 6). Both total (Fig. 6A) and methyl-branched (Fig. 6B) HC amounts evidenced a quite significant decrease but unexpectedly, the straight chain HC showed a significant increase in the RPRC000123-silenced specimens compared to controls (Fig. 6C). This result led us to check the transcript expression of the genes coding for the Rhodnius CYP4G enzymes (RPRC003227 and RPRC013847), which are involved in the final step of hydrocarbons formation (Qiu et al., 2012; Balabanidou et al., 2016). Both genes were significantly overexpressed in the RPRC000123silenced specimens compared to controls (data not shown), possibly due to an alteration in the regulation of the CYP4G transcript expression. This evidence might explain the increase in the straight chain $\mathrm{HC}$ content, likely associated to specific mobilization of straight chain LCFA that represent the bulk of integument fatty acids mostly palmitic and oleic acids- and are mainly stored as acylglycerols in the integument (Juárez \& Brenner, 1985). Also, the increase in the straight chain HC content could be attributed to additional non-integumental LCFA incorporation into the HC biosynthetic pathway. Wicker-Thomas et al. (2015) suggested that LCFA synthesis in the fat body may contribute to the pool of fatty acids used for $\mathrm{HC}$ biosynthesis in the 
oenocytes. The overexpression of the CYP4G genes might be a physiological compensatory mechanism to produce $\mathrm{HC}$ from an altered pool of LCFA precursors. Previous studies in mammals have shown that silencing the FASN1 gene affected the expression of a large number of genes from several metabolic pathways (Knowles \& Smith, 2007). Also, the up-regulation of genes involved in cell cycle arrest and apoptotic pathways was also observed, which might explain the death of the insects few days after moult, suggesting that other pathways were affected in addition to the lipid barrier (Fig. 7).

The results obtained after RPRC000123 silencing point out that the FASN encoded by this gene contributes to the waterproofing properties of the $R$. prolixus cuticle. The significant decrease in the methyl-branched $\mathrm{HC}$ amounts, associated to a lethal phenotype, strongly suggests that these components are the major responsible for regulating water balance, although straight chains represent the bulk of the cuticle $\mathrm{HC}$ (Juárez et al., 2001), and evidence a significant increase in RPRC000123-silenced insects. The participation of cuticle $\mathrm{HC}$ in waterproofing is related to its composition. Methyl-branched $\mathrm{HC}$, with lower melting points than straight chain $\mathrm{HC}$ of the same carbon number, have been proposed to have a major role in maintaining the barrier against evaporative water loss (Gibbs \& Pomonis, 1995). Using FASN silencing and desiccation assays in two Drosophila species, Chung et al. (2014) showed a direct correlation between the ability to survive under desiccation stress and the amount of methyl-branched HC.

Further studies are needed to address the relevance of the FASN3 in the general physiology and to explore its role in other pathways that use LCFA as precursors. Also, considering the selective lethality of FASN3 silencing, it may be interesting to clarify what physiological mechanisms essential for insect life FASN3 is involved in. Additionally, finding potential targets for RNAi-based vector control are expected goals to help contribute improving current vector control strategies. 


\section{Experimental procedures}

Insects

$R$. prolixus fourth- and fifth-stage nymphs were used for experimental procedures.

Third-stage nymphs were provided by the Servicio Nacional de Chagas (Córdoba, Argentina), and maintained at our insectary under standard rearing conditions $\left(27^{\circ} \mathrm{C}\right.$, $45 \% \mathrm{RH}, 12 \mathrm{~h}$ light : $12 \mathrm{~h}$ dark photoperiod) until further use. Insects were fed on rat blood once per development stage. All animal care and laboratory experimental protocols were carried out following the INIBIOLP Animal Welfare Assurance No. A5647-01, the AVMA Animal Welfare Policies and AVMA Guidelines on Euthanasia (https://www.avma.org/kb/policies/pages/default.aspx, https://www.avma.org/KB/Policies/Documents/euthanasia.pdf, last accessed January 12, 2019).

\section{Bioinformatic analyses}

FASN domains in RPRC000123, RPRC000269 and RPRC002909 protein sequences were detected and analysed by searching on the InterPro database (https://www.ebi.ac.uk/interpro/). Gene structure was obtained using the Gene Structure Display Server 2.0 (http://gsds.cbi.pku.edu.cn/). Phylogenetic analysis was performed on MEGA software (Tamura et al., 2013). FASN protein sequences of $R$. prolixus and other selected insect species were aligned using the ClustalW algorithm included in the software and then, a phylogenetic tree was constructed using the maximum likelihood method (Felsenstein, 1981), applying the LG model with gamma distribution for site substitutions. A bootstrap analysis was performed to test tree nodes' robustness, setting 1000 replications.

RNA extraction and cDNA synthesis

Ten days old fifth-stage nymphs were fed ad libitum and two weeks later, they were anaesthetised on ice, placed on a Petri dish, and their tissues and organs (brain, fat 
body, integument, Malpighian tubules, midgut and salivary glands) were dissected in cold saline solution $(0.9 \% \mathrm{NaCl})$ under a Zeiss Stemi 305 stereomicroscope (Carl Zeiss, Oberkochen, Germany) using appropriate forceps and scissors. Tissues and organs were homogenised separately with a rotor-stator homogeniser (Glas-Col, Terre Haute, USA) and total RNA was extracted using TRIzol Reagent, following manufacturer's instructions. Total RNA was quantitated on a Nanodrop 2000c spectrophotometer (Thermo Scientific, Wilmington, USA) and its quality assessed on a $1 \%(\mathrm{w} / \mathrm{v})$ agarose gel electrophoresis, stained with ethidium bromide. Single stranded cDNA was synthesised from $1 \mu \mathrm{g}$ total RNA using the iScript cDNA synthesis kit (BioRad, Hercules, USA), in $20 \mu$ l reaction volumes, according to manufacturer's recommendations. The temperature programme for reverse transcription was $5 \mathrm{~min}$ at $25^{\circ} \mathrm{C}, 20 \mathrm{~min}$ at $46^{\circ} \mathrm{C}$, and $1 \mathrm{~min}$ at $95^{\circ} \mathrm{C}$ to stop the reaction.

\section{Reverse transcription quantitative $P C R$}

FASN gene expression in different tissues and in silenced versus control insects was measured by RT-qPCR using the iQ SYBR Green Supermix (Bio-Rad, Hercules, USA), on a StepOnePlus Real-Time PCR system (Applied Biosystems, Foster City, USA). RT-qPCR reactions contained $5 \mu$ Sybr Green supermix, $4 \mu$ l cDNA diluted 10-fold, 0.4 $\mu \mathrm{l}$ of each primer (200 nM concentration), and Milli-Q water in a total volume of $10 \mu \mathrm{l}$. Temperature programme for RT-qPCR was: one initial denaturation cycle for $3 \mathrm{~min}$ at $95^{\circ} \mathrm{C}$, followed by 40 cycles at $95^{\circ} \mathrm{C}$ for $15 \mathrm{~s}$ and $60^{\circ}$ for $60 \mathrm{~s}$. In order to check for unspecific product formation, a post-amplification melting curve analysis was performed under the following temperature programme: $30 \mathrm{~s}$ at $95^{\circ} \mathrm{C}, 30 \mathrm{~s}$ at $65^{\circ} \mathrm{C}$, then an increase in temperature to $95^{\circ} \mathrm{C}$ for 30 s with measurement of total SYBRGreen fluorescence every $0.5^{\circ} \mathrm{C}$. $\beta$-actin and EF-1 (elongation factor 1 ) were used as reference genes for normalisation of FASN gene expression, according to Majerowicz et al. (2011). The stability of the reference genes was tested using the GeNorm algorithm (Vandesompele et al., 2002). Primer sequences were designed with the 
GeneRunner v. 3.1 software (http://www.generunner.net/) and are shown in Supporting information (Table S1). Four biological replicates (5 insects each) were used, and two technical replicates of each sample were performed in order to check for pipetting errors. No template and no reverse transcriptase controls were performed to detect primer dimer formation and contamination with genomic DNA. Primer efficiencies were determined using 5-fold dilution series (1:5 down to 1:3125) from pooled cDNA to generate standard curves for each primer pair. The relative quantitation of the FASN genes in comparison to the reference genes was calculated according to Hellemans et al. (2007) and expressed as the normalised relative quantity (NRQ) of each one.

\section{FASN gene silencing by RNA interference}

Double-stranded RNA was synthesised for each of the $R$. prolixus FASN genes (RPRC000123, RPRC000269, and RPRC002909) using the MEGAscript RNAi kit (Ambion, Austin, USA), following manufacturer's recommendations. FASN gene DNA templates, which served for dsRNA synthesis, were amplified by PCR using primers containing the T7 RNA polymerase promoter at their 5' end. Primers were designed using the GeneRunner software; primer sequences are detailed in Supporting Information Table S1. PCRs were performed using the Platinum Taq DNA Polymerase (Invitrogen, Carlsbad, USA) in total volumes of $50 \mu$ l, according to manufacturer's instructions. Temperature programme for PCR reactions was: 2 min at $94^{\circ} \mathrm{C}$, followed by 35 cycles of $30 \mathrm{~s}$ at $94^{\circ} \mathrm{C}, 30 \mathrm{~s}$ at $55^{\circ} \mathrm{C}$, and $1 \mathrm{~min}$ at $72^{\circ} \mathrm{C}$. DNA and dsRNA concentrations were measured on a Nanodrop 2000c spectrophotometer, and their quality and size verified on a $1 \%(\mathrm{w} / \mathrm{v})$ agarose gel electrophoresis. One microliter of dsRNA solution $(1 \mu \mathrm{g} / \mu \mathrm{l})$, either dsRPRC000123, dsRPRC000269 or dsRPRC002909, was injected in the ventral abdomen of unfed fourth-stage nymphs ( $n=150$ each) previously anaesthetised on ice, using a $10 \mu$ l Hamilton syringe fitted with a 33-gauge needle (Hamilton Company, Reno, USA). Insects used as controls of the silencing experiments were injected with the same amount of the negative control provided with 
the kit $(D$. melanogaster nautilus gene, a transcription factor involved in muscle formation in embryos, referred herein as dsControl; $n=150)$. Insects were fed ad libitum 3 days post injection, and five days post feeding a sample of each condition was used to check the efficiency of gene silencing by RT-qPCR.

\section{Integument fatty acid and hydrocarbon analyses}

The abdominal integuments of both dsControl- and dsRPRC000123-injected fourthstage nymphs were dissected 14 days post injection as described in a previous section and stored separately at $-70^{\circ} \mathrm{C}$ in aluminium foil. For fatty acid analysis, the integuments ( 3 replicates of 5 insects each for each sample) were homogenised using a glass homogeniser, and their lipids extracted with chloroform:methanol (2:1). Lipids were saponified with $10 \% \mathrm{KOH}$ in methanol $(\mathrm{v} / \mathrm{v})$ at $80^{\circ} \mathrm{C}$ for $60 \mathrm{~min}$, and fatty acids transesterified to methyl esters with $20 \% \mathrm{BF}_{3}$ in methanol $(\mathrm{v} / \mathrm{v})$ for 90 min at $65^{\circ} \mathrm{C}$. FAMEs were quantitated by CGC on a Hewlett-Packard 6890 gas chromatograph (Hewlett Packard, Wilmington, USA) coupled to a flame ionization detector (FID), and fitted with an OmegaWax 250 capillary column (30 m long x $0.25 \mathrm{~mm}$ internal diameter x $0.25 \mu \mathrm{m}$ stationary phase) (Supelco, Bellefonte, USA (Juárez et al., 2000). Injection port was operated at $260^{\circ} \mathrm{C}$ in splitless mode and the FID was held at $300^{\circ} \mathrm{C}$. Helium was used as mobile phase (2 $\mathrm{ml} / \mathrm{min})$. Temperature programme for the CGC run was set as follows: $50^{\circ} \mathrm{C}$ for $1 \mathrm{~min}$ (initial), from $50^{\circ} \mathrm{C}$ to $175^{\circ} \mathrm{C}$ at a rate of $50^{\circ} \mathrm{C} / \mathrm{min}$ (ramp 1), from $175^{\circ} \mathrm{C}$ to $260^{\circ} \mathrm{C}$ at a rate of $3^{\circ} \mathrm{C} / \mathrm{min}$ (ramp 2), and then held at $260^{\circ} \mathrm{C}$ for 10 min. FAME peak areas were calculated for each chromatogram (HP Chem Station, Hewlett Packard) and compared to those of 11 -eicosenoic acid (Nu Check Prep, Elysian, USA), which was used as internal standard for quantitation. Identification of FAMEs was performed by CGC-MS using a Hewlett-Packard 6890 gas chromatograph (Hewlett Packard, Wilmington, USA) coupled to an Agilent 5975C VL MS detector (Agilent, Santa Clara, USA). CGC conditions were the same as described for FAME quantitation; the MS detector was set up as follows: ionizing energy $70 \mathrm{eV}$, the ion 
source, transfer line and quadrupole were held at $230^{\circ} \mathrm{C}, 260^{\circ} \mathrm{C}$ and $150^{\circ} \mathrm{C}$, respectively. FAMEs were identified by library search, analysis of their fragmentation pattern and comparison with standards similarly run. For hydrocarbon analysis, the integuments ( 3 replicates of 5 insects each for each condition) were submerged $48 \mathrm{~h}$ in redistilled hexane in order to extract total lipids. Hexane volume was reduced under a nitrogen stream, and hydrocarbons were purified by adsorption chromatography on a mini-column (10 mm x 5 mm I.D.) of activated Biosil A (Bio-Rad, Richmond, USA), eluting with $4 \mathrm{ml}$ of redistilled hexane. Hydrocarbons were quantitated by CGC-FID on a Hewlett-Packard 6890 gas chromatograph fitted with a Zebron ZB-5HT Inferno capillary column (30 m long x $0.25 \mathrm{~mm}$ internal diameter $\times 0.25 \mu \mathrm{m}$ stationary phase) (Phenomenex, Torrance, USA). Injection port (splitless mode) and FID were set at $360^{\circ} \mathrm{C}$. Helium was used as mobile phase $(2 \mathrm{ml} / \mathrm{min})$. Temperature programme for CGC was set up as follows: $50^{\circ} \mathrm{C}$ for $1 \mathrm{~min}$ (initial), from $50^{\circ} \mathrm{C}$ to $200^{\circ} \mathrm{C}$ at a rate of $50^{\circ} \mathrm{C} / \min \left(\right.$ ramp 1 ), from $200^{\circ} \mathrm{C}$ to $360^{\circ} \mathrm{C}$ at a rate of $7^{\circ} \mathrm{C} / \mathrm{min}$ (ramp 2), and then held at $360^{\circ} \mathrm{C}$ for $20 \mathrm{~min}$. Hydrocarbon peak areas were calculated for each chromatogram (HP Chem Station, Hewlett Packard) and compared to docosane (Sigma-Aldrich, San Luis, USA) peak areas, which was used as internal standard for quantitation. Identification of hydrocarbons was performed by CGC-MS in the same equipment as described above. Chromatographic conditions were the same as described for CGCFID and the MS detector was operated as follows: ionizing energy $70 \mathrm{eV}$, the ion source, transfer line and quadrupole were held at $280^{\circ} \mathrm{C}, 360^{\circ} \mathrm{C}$ and $150^{\circ} \mathrm{C}$, respectively. Hydrocarbons were identified according to Juárez et al. (2001).

\section{High humidity rescue bioassay}

Both dsControl- and dsRPRC000123-injected fourth-stage nymphs ( $\mathrm{n}=45$ each condition) were placed into a humid chamber ( $27^{\circ} \mathrm{C}, 96 \%$ relative humidity) 3 days before moulting to fifth nymph stage, and mortality was daily recorded. 


\section{Statistical analyses}

Statistical significance was assessed using a one-way ANOVA followed by Tukey's multiple comparison test or an unpaired Student's t-test, depending on the number of experimental groups under analysis. Graph Pad Prism 5.01 software (GraphPad Software, San Diego, USA) was used for statistical analyses.

\section{Acknowledgements}

We would like to thank Dr. María José Villalobos for helping with dissections, Dr. Raúl Stariolo for insect provision, Lic. Sergio Mijailovsky and Lic. Belén Gastaca for their assistance in chromatographic analyses, Dr. Gareth Weedall for his advice on genomic analysis, and also Cecilia Fuse and Facundo Bozzolo for insect rearing. This work was supported by grant PIP0390 from the National Scientific and Technical Research Council (CONICET). MPJ and GMCF are members of the CONICET Researcher's Career, Argentina. The authors declare no conflicts of interest with this work.

\section{References}

Arrese, E., Canavoso, L., Jouni, Z., Pennington, J., Tsuchida, K. and Wells, M. (2001) Lipid storage and mobilization in insects: current status and future directions. Insect Biochem Mol Biol 31: 7-17.

Balabanidou, V., Kampouraki, A., MacLean, M., Blomquist, G., Tittiger, C., Juárez, M. et al. (2016) Cytochrome P450 associated with insecticide resistance catalyzes cuticular hydrocarbon production in Anopheles gambiae. Proc Natl Acad Sci 113: 9268-9273.

Billeter, J., Atallah, J., Krupp, J., Millar, J.G. and Levine, J. (2009) Specialized cells tag sexual and species identity in Drosophila melanogaster. Nature 461: 987-991.

Blomquist, G. and Bagnères, A-G. (2010) Introduction: History and overview of insect hydrocarbons. In Insect Hydrocarbons: Biology, Biochemistry, and Chemical Ecology (Blomquist, G.J. and Bagnères, A-G., eds), pp. 3-18. Cambrige 
University Press, Cambridge.

Calderón-Fernández, G., Moriconi, D., Dulbecco, A. and Juárez, M. (2017)

Transcriptome Analysis of the Triatoma infestans (Hemiptera: Reduviidae)

Integument. J Med Entomol 54: 1531-1542.

Chung, H., Loehlin, D., Dufour, H., Vaccarro, K., Millar, J. and Carroll, S. (2014) A single gene affects both ecological divergence and mate choice in Drosophila. Science 343: 1148-1151.

De Renobales, M., Woodin, T. and Blomquist, G. (1986) Drosophila melanogaster fatty acid synthetase: Characteristics and effect of protease inhibitors. Insect Biochem 16: 887-894.

De Schrijver, E., Brusselmans, K., Heyns, W., Verhoeven, G. and Swinnen, J. (2003) RNA interference-mediated silencing of the fatty acid synthase gene attenuates growth and induces morphological changes and apoptosis of LNCaP prostate cancer cells. Cancer Res 63: 3799-3804.

Felsenstein, J. (1981) Evolutionary trees from DNA sequences: a maximum likelihood approach. J Mol Evol 17: 368-376.

Finck, J., Berdan, E., Mayer, F., Ronacher, B. and Geiselhardt, S. (2016) Divergence of cuticular hydrocarbons in two sympatric grasshopper species and the evolution of fatty acid synthases and elongases across insects. Sci Rep 6: 33695.

Gibbs, A. (1998) Water-Proofing Properties of Cuticular Lipids. Am. Zool. 38: 471-482.

Gibbs, A. and Pomonis, J. (1995) Physical properties of insect cuticular hydrocarbons: The effects of chain length, methyl-branching and unsaturation. Comp Biochem Physiol Part B Biochem Mol Biol 112: 243-249.

Gu, P., Welch, W. and Blomquist, G. (1993) Methyl-branched fatty acid biosynthesis in the German cockroach, Blatella germanica: Kinetic studies comparing a microsomal and soluble fatty acid synthetase. Insect Biochem Mol Biol 23: 263271.

Gu, P., Welch, W., Guo, L., Schegg, K. and Blomquist, G. (1997) Characterization of a 
novel microsomal fatty acid synthetase (FAS) compared to a cytosolic FAS in the housefly, Musca domestica. Comp Biochem Physiol - B Biochem Mol Biol 118B: 447-456.

Hadley, N. (1994) Water Relations of Terrestrial Arthropods. Academic Press, San Diego. 356 p.

Hellemans, J., Mortier, G., De Paepe, A., Speleman, F. and Vandesompele, J. (2007) qBase relative quantification framework and software for management and automated analysis of real-time quantitative PCR data. Genome Biol 8: R19.

Juárez, M. (1994) Inhibition of cuticular lipid synthesis and its effect on insect survival. Arch. Insect Biochem Physiol 25: 177-191.

Juárez, M. (1995) The effect of sublethal doses of insecticides on Triatoma infestans lipid synthesis. Pestic Biochem Physiol 52: 81-87.

Juárez, M. (1996) Composición de efecto insecticida, método de aplicación. Patent \#249780, Argentina.

Juárez, M. and Brenner, R. (1985) The epicuticular lipids of Triatoma infestans-II. Hydrocarbon dynamics. Comp Biochem Physiol 82B: 793-803.

Juárez, M. and Brenner, R. (1989) Fatty acid biosynthesis in the integument tissue of Triatoma infestans. Comp Biochem Physiol 93: 763-772.

Juárez, M. and Calderón-Fernández, G. (2007) Cuticular hydrocarbons of triatomines. Comp Biochem Physiol - A Mol Integr Physiol 147: 711-730.

Juárez, M., Ayala, S. and Brenner, R. (1996) Methyl-Branched Fatty Acid Biosynthesis in Triatoma infestans. Insect Biochem Mol Biol 26: 599-605.

Juárez, M., Blomquist, G. and Schofield, C. (2001) Hydrocarbons of Rhodnius prolixus, a Chagas disease vector. Comp Biochem Physiol - B Biochem Mol Biol 129: 733746.

Juárez, M., Chase, J. and Blomquist, G. (1992) A Microsomal Fatty Acid Synthetase from the Integument of Blattella Germanica Synthesizes Methyl-Branched Fatty 
Acids, Precursosrs to Hydrocarbon and Contact Sex Pheromone. Arch Biochem Biophys 293: 333-341.

Juárez, M., Crespo, R., Calderón Fernández, G., Lecuona, R. and Cafferata, L. (2000) Characterization and Carbon Metabolism in Fungi Pathogenic to Triatoma infestans, a Chagas Disease Vector. J Invertebr Pathol 76: 198-207.

Knowles, L. and Smith, J. (2007) Genome-wide changes accompanying knockdown of fatty acid synthase in breast cancer. BMC Genomics 8: 1-13.

Maier, T., Leibundgut, M. and Ban, N. (2008) The crystal structure of a mammalian fatty acid synthase. Science 321 : 1315-1322.

Majerowicz, D., Alves-Bezerra, M., Logullo, R., Fonseca-De-Souza, A., MeyerFernandes, J., Braz, G. et al. (2011). Looking for reference genes for real-time quantitative PCR experiments in Rhodnius prolixus (Hemiptera: Reduviidae). Insect Mol Biol 20: 713-722.

Majerowicz, D., Calderón-Fernández, G., Alves-Bezerra, M., De Paula, I., Cardoso, L., Juárez, M. et al. (2017) Lipid metabolism in Rhodnius prolixus: lessons from the genome. Gene 596: 27-44.

Martins, G., Guedes, B., Silva, L., Serrão, J., Fortes-Dias, C., Ramalho-Ortigão, J. et al. (2011) Isolation, primary culture and morphological characterization of oenocytes from Aedes aegypti pupae. Tissue Cell 43: 83-90.

Mesquita, R., Vionette-Amaral, R., Lowenberger, C., Rivera-Pomar, R., Monteiro, F., Minx, P. et al. (2015) Genome of Rhodnius prolixus, an insect vector of Chagas disease, reveals unique adaptations to hematophagy and parasite infection. Proc Natl Acad Sci 112: 14936-14941.

Parvy, J., Napal, L., Rubin, T., Poidevin, M., Perrin, L., Wicker-Thomas, C. et al. (2012) Drosophila melanogaster Acetyl-CoA-Carboxylase Sustains a Fatty AcidDependent Remote Signal to Waterproof the Respiratory System. PLoS Genet 8: e1002925.

Qiu, Y., Tittiger, C., Wicker-Thomas, C., Le Goff, G., Young, S., Wajnberg, E. et al. 
(2012) An insect-specific P450 oxidative decarbonylase for cuticular hydrocarbon biosynthesis. Proc Natl Acad Sci 109: 14858-14863.

Tamura, K., Stecher, G., Peterson, D., Filipski, A. and Kumar, S. (2013) MEGA6:

Molecular evolutionary genetics analysis version 6.0. Mol Biol Evol 30: 27252729.

Tan, Q., Liu, W., Zhu, F., Lei, C. and Wang, X. (2017) Fatty acid synthase 2 contributes to diapause preparation in a beetle by regulating lipid accumulation and stress tolerance genes expression. Sci Rep 7: 1-11.

Vandesompele, J., De Preter, K., Pattyn, F., Poppe, B., Van Roy, N. ,De Paepe, A. et al. (2002) Accurate normalization of real-time quantitative RT-PCR data by geometric averaging of multiple internal control genes. Genome Bio/ 3: 1-11.

Wicker-Thomas, C., Garrido, D., Bontonou, G., Napal, L., Mazuras, N., Denis, B. et al. (2015) Flexible origin of hydrocarbon/pheromone precursors in Drosophila melanogaster. J Lipid Res 56: 2094-2101.

Wigglesworth, V. (1933) The Physiology of the Cuticle and of Ecdysis in Rhodnius prolixus (Triatomidae, Hemiptera); with special reference to the function of the oenocytes and of the dermal glands. J Cell Sci 76: 269-318.

Wigglesworth, V. (1945) Transpiration through the cuticle of insects. J Exp Biol 21: 97114.

Wigglesworth, V. (1975) Incorporation of lipid into the epicuticle of Rhodnius (Hemiptera). J Cell Sci 19: 459-85.

Zhou, Y., Buijs, N., Zhu, Z., Qin, J., Siewers, V. and Nielsen, J. (2016) Production of fatty acid-derived oleochemicals and biofuels by synthetic yeast cell factories. Nat Commun 7: 11709 .

\section{Supporting Information}

Table S1. Primers used in this study. 


\section{Figure legends}

Figure 1. Expression pattern of FASN genes in different tissues and organs of $R$. prolixus fifth-stage nymphs. The transcription levels of $R P R C 000123$ (A), RPRC002909 (B) and RPRC000269 (C) genes were measured by RT-qPCR in the Malpighian tubules, midgut, fat body, integument, salivary glands and brain of $R$. prolixus. For each gene, the normalised relative quantity (NRQ) was estimated using $\beta$-actin and elongation factor 1 (EF-1) as reference genes. Error bars represent SE of the means of 4 biological replicates (5 insects each). Asterisks indicate significant differences determined by a One-Way ANOVA followed by a Tukey multiple comparison test ( $P$ $<0.05)$. Significant expression levels were detected in the integument for genes $R P R C 000123$ and RPRC002909, and in the fat body for gene RPRC000269.

Figure 2. Genomic and protein organization of the $R$. prolixus FASN genes $R P R C 000123$ (A), RPRC002909 (B), and RPRC000269 (C). For each one, the genomic (up) and the protein structures (down) are shown. Green and black numbers indicate exon and intron size in bp, respectively. Protein domains are marked with different colour boxes. Animal FASNs contain 7 protein domains (from the amino terminal to the carboxyl terminal are: KS, MAT, DH, ER, KR, ACP and TE); some of them are missing in the R.prolixus FASNs. The protein RPRC002909 has two predicted transmembrane domains (tm) immediately before the ER domain.

Figure 3. Phylogenetic analysis of $R$. prolixus FASN genes. Sequence codes correspond to access numbers in GenBank. The dendrogram was constructed using the maximum likelihood method with 1000 bootstrap replications. Numbers at nodes indicate the bootstrap support (\%). R. prolixus sequences are highlighted in coloured squares. Insect sequences included correspond to those insects with oenocytes associated with the integument tissue; they were separated into two main groups, 
Cluster I and Cluster II. FASN sequences of S. mimosarium, C. elegans, P. vannamei, I. scapularis and $H$. sapiens were used as outgroups.

Figure 4. $R$. prolixus fifth-stage nymph phenotypes resulting from FASN genes silencing. Ten days old fourth-stage nymphs were injected with dsRNA of each FASN gene (dsRPRC000123, dsRPRC000269 or dsRPRC002909), or with dsRNA of the control provided by the silencing kit (dsControl). Insects were maintained at $27^{\circ} \mathrm{C}$ and $45 \% \mathrm{RH}$ and fed ad libitum 3 days post injection. They were observed daily until moult to fifth nymph stage. dsRPRC000269- and dsRPRC002909-injected insects survived normally after moulting, similarly to dsControl-injected insects. dsRPRC000123injected insects died immediately after moulting, with incomplete cuticle sclerotization and pigmentation.

Figure 5. Effect of RPRC000123 silencing on the integument fatty acids of $R$. prolixus nymphs. Fourth-stage nymphs were injected with either dsRPRC000123 or dsControl solutions and 14 days later, the integuments were excised, and fatty acids analysed by CGC-MS. LCFA are expressed as micrograms $(\mu \mathrm{g})$ per integument. Total $(A)$, straight chain (B) and methyl-branched (C) LCFA were significantly reduced by $36.08 \%$, $35.80 \%$ and $73.18 \%$, respectively in RPRC000123-silenced nymphs compared to controls. Error bars represent SE of the means of 3 biological replicates ( 5 insects each). Asterisks indicate significant differences between control and RPRC000123silenced nymphs determined by an unpaired one-tailed Student's t test ${ }^{* *}, P<0.01$; ***, $\mathrm{P}<0.001)$.

Figure 6. Effect of $R P R C 000123$ silencing on the integument $\mathrm{HC}$ of $R$. prolixus nymphs. Fourth-stage nymphs were injected with either dsRPRC000123 or dsControl solutions; 14 days later, the integuments were excised, and HC analysed by CGC-MS. $\mathrm{HC}$ are expressed as nanograms (ng) per integument. Total (A), and methyl-branched 
(B) $\mathrm{HC}$ were significantly reduced by $23.42 \%$ and $46.38 \%$, respectively in RPRC000123-silenced nymphs compared to controls; straight chain HC showed a significant increase (126.22\%) (C). Error bars represent SE of the means of 3 biological replicates (5 insects each). Asterisks indicate significant differences between control and RPRC000123-silenced nymphs determined by an unpaired one-tailed Student's t test $\left({ }^{* *}, \mathrm{P}<0.01 ;{ }^{* *}, \mathrm{P}<0.001\right)$.

Figure 7. Survival of control and RPRC000123-silenced fifth-stage nymphs after 3 days of incubation at different relative humidity conditions. When reared under our standard insectary conditions $\left(27^{\circ} \mathrm{C}, 45 \% \mathrm{RH}\right)$, dsRPRC 000123 -injected fourth-stage nymphs moulted to fifth stage and died immediately after, whilst dsControl-injected nymphs survived normally after moulting $(A)$. When both insect groups were placed into a humid chamber 3 days prior to moulting $\left(27^{\circ} \mathrm{C}, 96 \% \mathrm{RH}\right)$, the $R P R C 000123-$ silenced nymphs survived for about 48-72 $\mathrm{h}$ after moult, whilst the control nymphs remained alive ( $n=45$ for each condition) (B). Asterisks indicate a significant difference between control and RPRC000123-silenced nymphs determined by an unpaired onetailed Student's t test $(P<0.001)$. 

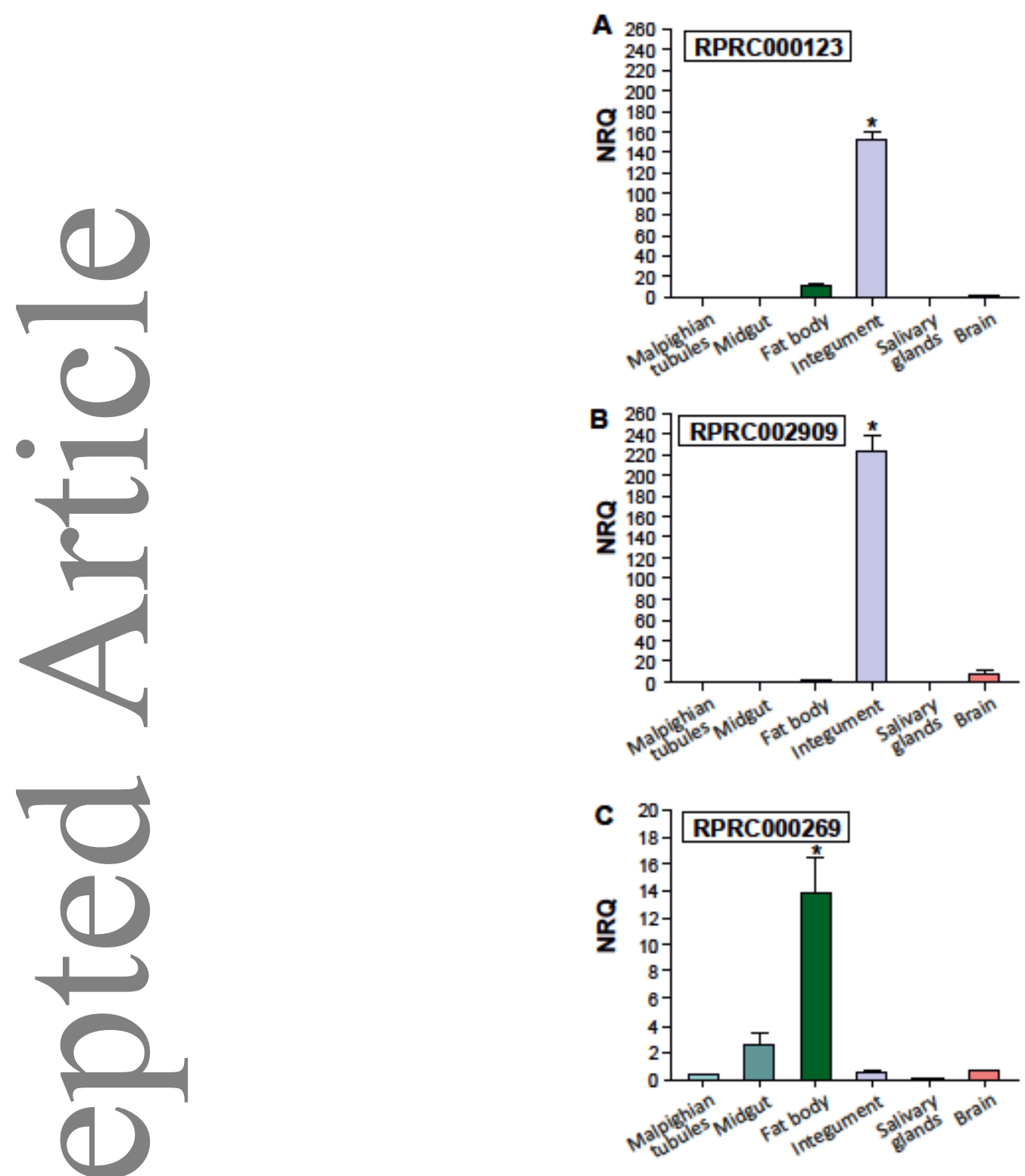


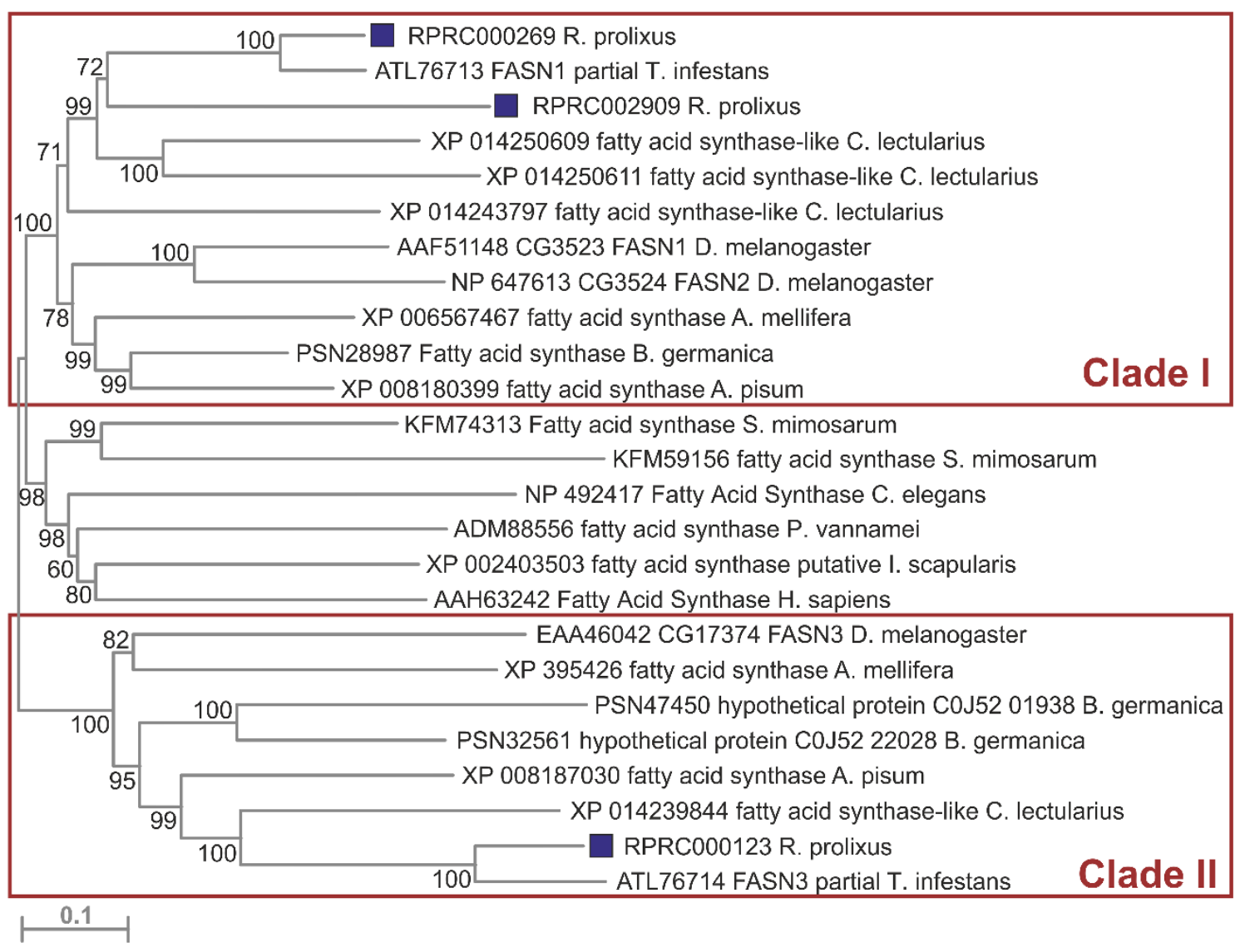

This article is protected by copyright. All rights reserved. 

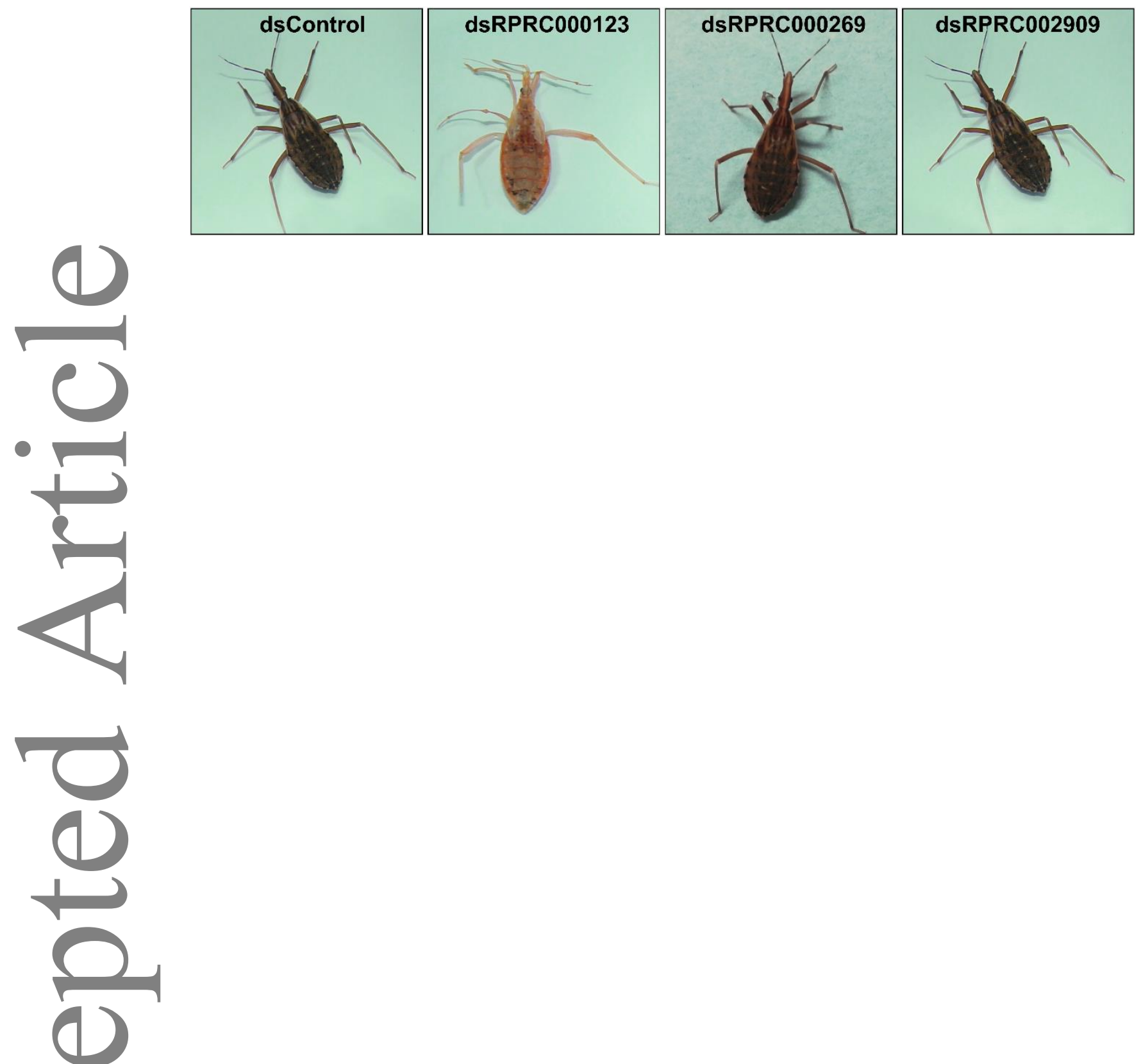

$P$

( )

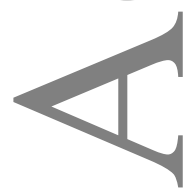

This article is protected by copyright. All rights reserved. 

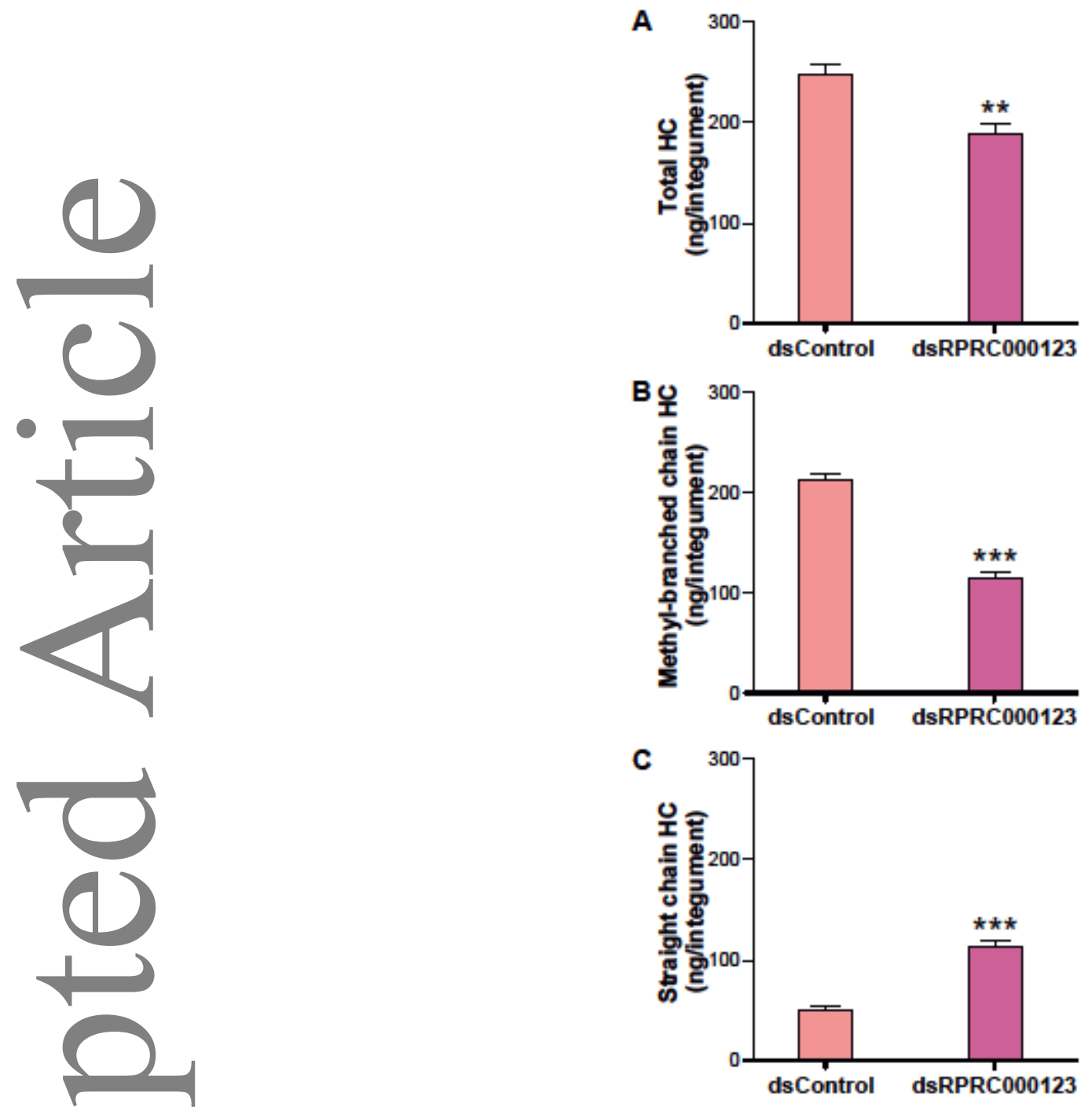

This article is protected by copyright. All rights reserved. 
